\title{
Coverage Area of a Localization Fixed Sensors Network System with the process of Triangulation
}

\author{
${ }^{1}$ Marios SFENDOURAKIS, ${ }^{2}$ MARIA ZAKYNTHINAKI, ${ }^{3}$ ERIETTA VASILAKI, \\ ${ }^{4}$ EMmanUEl ANTONIDAKIS, ${ }^{5}$ RAJAgOPAL NiLAVALAN \\ ${ }^{1}$ Brunel University- Dept of Electronic and Computer Engineering ,UB8 3PX Kingston Lane, \\ Uxbridge Middlesex, United Kingdom. \\ ${ }^{2}$ Hellenic Mediterranean University - Department of Electronic Engineering, Romanou 3, Chania, \\ Greece. \\ ${ }^{3}$ Hellenic Mediterranean University - Department of Electronic Engineering, Romanou 3, Chania, \\ Greece. \\ ${ }^{4}$ Hellenic Mediterranean University - Department of Electronic Engineering, Romanou 3, Chania, \\ Greece \\ ${ }^{5}$ Brunel University- Dept of Electronic and Computer Engineering ,UB8 3PX Kingston Lane, \\ Uxbridge Middlesex, United Kingdom.
}

\begin{abstract}
This paper presents a novel work on localization of transmitters using triangulation with sensors at fixed positions. This is achieved when three or more sensors cover the whole area, a factor which enables the system to perform localization via triangulation. The network needs to keep a high detection rate which, in most cases, is achieved by adequate sensor coverage. Various tests using various grids of sensors have been carried out to investigate the way the system operates in different cases using a lot of transmitters. Detection complexity is tackled by finding the optimal detecting sensor radius in order the network to continue operate normally. The coverage quality changes in the area of interest and the network is able to detect new transmitters that might enter it's area. It is also shown that as the number of transmitters increases the network keeps its high performance by using additional groups of sensors in a sub-region area of that of interest. This way, even when the network is saturated by many transmitters in one region, new transmitters can still be detected.
\end{abstract}

Keywords: Fixed Sensors Network, Triangulation, Localization, Sensor Blindness, Detection Range.

Received: December 4, 2020. Revised: May 8 2021. Accepted: May 31, 2021. Published: June 15, 2021.

\section{Introduction}

In this research we are providing a solution to the problem of localization with the process of triangulation. The process of positioning with triangulation is currently under research regarding a large number of applications. In our case we use various grids of fixed Sensors - SRs in order to detect possible transmitters (TRs) which might enter an area. In the last decade, coverage was a fundamental research issue in WSNs. It was considered to be the measure of QoS for the sensing function of a sensor network [1]. The sensors are in fixed and known positions. So, we don't have to process the SRs positions which is also an issue of extensive research. The system has to process its state and acquire possible problematic areas of nocoverage, make relevant changes in order to increase the detection performance rate, in case a new TR enters its area of coverage. One of the changes that a network has to execute is to find the optimal radius of detection which will enable it to preserve a high detection rate. That means that from an initial state 1 to a new state 2 etc, the network has to perform a kind of adaptation to the new 
circumstances and change its parameters. That might also include additional groups of SRs to be used in order to achieve TRs detection with triangulation. In this paper, we'll also show how additional groups of SRs activated close to a problematic area will increase the network performance. We assume that the visualization of changes in the network performance, plays a significant role in order to tackle problematic areas of no coverage.

\section{Related work}

In last decade authors are exploring thoroughly localization systems and applications of learning algorithms related with localization based on radio frequency and inertial sensors. It was noted that localization issues related with availability, scalability, privacy and security will address new challenges in upcoming $5 \mathrm{G}$ communication industry trends [2]. Furthermore, as localization and positioning research in WSNs is more and more deepening a wide range of applications, such as target tracking habitat monitoring and intelligent environments require precise positioning of the global coordinate system in order to provide meaningful information and propagate efficiently data over the network [3]. Brief reviews on enabling wireless technologies for localization in indoor tracking and positioning systems (IPSs) and localization techniques for WSNs are available in [4], [5], and [6] where it can be seen the significance of SRs topology network coverage and their impact on network performance. Also last years, the researchers and engineers attention were attracted in developing systems for tracking and navigation due to the high consumer penetration of sensor-rich mobile devices and the ubiquity of WSNs systems for localization. These systems span different application domains. So, various systems appeared, from emergency call positioning, such as E911 in the U.S.A. and E112 in EU to customer-centric location-based services, such as mobile advertising, behavioural retail analytics to resource allocation [7]. Moreover, a new era of crowd sensing WSNs is now appearing. This happens as modern mobile devices have higher processing capabilities in combination with a wide availability of sensing modules. Also, and as networks of SRs appear more and more in our daily life in smart homes, smart cars etc, this results to a new type of wide-area WSN-crowd-based communication technologies paving the way to new and innovative applications [8]. An extended analysis of coverage problems is provided in [9]. Nowadays, as a great number of sensors - SRs forming a network are deployed in an area collecting data, a targets position is acquired by transmitted data processing. Nevertheless, and as more and more localization systems are developed based on different approaches and techniques of positioning, a localization technique appropriate for an application might be completely inappropriate for another. Meaning that a localization system used for example for determining a cars position during its movement might be completely different from an indoor WSN system that deals with interactive tour guidance. Although both systems require localization information the algorithms used and the philosophy of operation of the systems are completely different [10]. Additionally, position information is crucial in many other location-based applications. Among them there are the domain of autonomous logistics, search-and-rescue services and security of buildings [11]. Currently, localization is an important technique in WSNs and recently a great number of researchers showed their interest in localization through WSNs. Also, indoor localization by using WSNs became a reality by the advancement in electronics in wireless communications [12]. Also, localization is used for many other purposes, including surveying, navigation, metrology, astrometry, model rocketry, binocular vision and gun direction of weapons [13]. Furthermore, WSNs offers a variety of applications and solutions. In order to achieve network performance optimization a counterbalance between power consumption and area coverage is required. Also, network overage optimization requires nodes topology in such a way that maximum area coverage is achieved with a minimum number of nodes [14]. Moreover in current research, more and more researchers seek to solve the problem of localization for various applications and systems, and network coverage is an issue of prime importance. As far as network coverage is concerned it is categorized in three types: Area coverage, target coverage and 
barrier coverage [15-22]. Network coverage in WSNs is defined as each point in the ROI is covered by at least one SR, $(k \geq 1)$ [23].Thus the performance of WSNs is strongly related with the quality of area coverage and many researchers seek for ways to optimize network performance. Also, the type of network topology followed is affecting network performance and has to be examined thoroughly [24-26]. SRs relocation problem for RSSI-based target localization in WSNs was examined in [27]. Problem tackling included various methods and the movement of SRs to relative points of interest in the ROI with satisfactory results. Moreover, the practical use in a great deal of special domains which included military, environmental, the health sector etc. was examined in [28]. The outcome of this examination was that WSNs technology might lead to innovative ways of tackling problems compared with traditional methods previously used. In [29] authors presented a sensor-fusion-system for automatically tracking sheep localization and behavior. The proposed system was able to detect sheep standing and resting behaviours and track individual animal's location in the production environment. $\mathrm{Yu}$ et al. in [30] proposed a method of low-speed impact (LVI) monitoring system. The presented methodology is reducing the average error of positioning proving the effective means of LVI positioning. Target localization appears also in vehicles that might act a SRs with a relative deployment. Another issue that affects seriously a WSNs area coverage and network performance is the area coverage holes. A great deal of research nowadays focuses to solve the problem of coverage holes in the ROI. The performance of WSNs in terms of area coverage is a critical issue that needs extra attention and research. In [31] seeking for the maximization of area coverage and k-coverage, a genetic algorithm and a particle swarm optimization algorithm were used. Although the results were promising it was mentioned that there are many other problems that generally obstruct the network operation either directly or indirectly affecting the optimization of performance. For that reason coverage performance optimization should be pursued by taking into consideration energy conservation and SRs connectivity preservation. In [32] the optimal deployment of $n$ vehicles with circular formation for 2D bearingsonly multi-target localization is presented. In order to track the assigned target, $\mathrm{n}$ mobile vehicles are first distributed randomly in the region of targets, then the vehicles are redeployed and forced to be gathered around the assigned target with circular formation to estimate the target location. An explicit formula of GDOP is used to evaluate the localization accuracy with different sensor-target geometries. Also, an efficient optimal deployment algorithm of vehicles is developed for multi-target localization. Another issue that affects seriously the WSN coverage area and the network performance is the coverage holes. Authors in [33] proposed a NLCHR (Neighbor node Location-based Coverage Hole Recovery) that recovers coverage holes by adding mobile SR nodes at an optimal location using a minimum amount of resources in exchange of information with neighbor boundary nodes. The proposed method adds the mobile SR node to the optimal location based on the location of two adjacent hole boundary nodes, and reliably recovers the entire coverage hole through an iterative round. In [34], the coverage model of RSSI-based positioning technology was explored, and the RSSIbased positioning was enhanced through the detection and recovery of coverage holes. It is also proposed an algorithm inspired by Voronoi tessellation and Delaunay triangulation to detect and recover coverage holes with very good results. In [35] a new game theory approach based on reinforcement learning to recover Coverage Holes in a distributed way is proposed. For the formulated potential game, SR nodes can recover Coverage Holes using only local acquaintances. Coverage gaps are reduced by combining node reposition and detection range adjustment by each sensor node. Unlike previous methods simulation results proved that the proposed method can sustain a high network coverage in the presence of random damage events. In [36] all the coverage holes in the network are identified and classified as closed hole or open holes with the CBHD method. With the CBHC method it is demonstrated that a coverage hole can be recovered efficiently by using the minimum number of additional SR nodes and with the minimum coverage area overlap. These hole detection and hole covering methods might be used effectively in 
any type of ROI to detect coverage holes, demarcate the boundary of network and heal the sensing coverage holes. Also, WSNs are often deployed for event detection and environmental monitoring. However, it is mentioned that their success in providing quality of service can only be ensured if the network does not have any sensing coverage holes. And frequently, the existence of sensing coverage holes is unavoidable due to various factors such as environmental disasters, random deployment and hardware failure of the SR node [37]. Authors in [38] have raised a few research queries which included the automated coverage control which is directly associated with the QoS of WSNs. Hole-clustering and hole-area estimation are also tightly bound with coverage and connectivity issues. Network coverage optimization is among the key factors in designing efficient algorithms and adequate SR deployment for WSNs. In [39] Gupta et al. proposed a meta-heuristic approach on the basis of Genetic Algorithm (GA) to identify the position of the SR nodes to be placed so that all target points are k-covered and nodes are $\mathrm{m}$ connected. The $k$-coverage of the targets, means that each of the target points must be covered by at least $k$ SR nodes. In this work it isn't analyzed network coverage performance deterioration due to existing targets and the issue of blindness. In current literature exists a great variety of different approaches for network coverage holes healing and recovery, holes identification and detection, aiming at diminishing the problem of Hole Coverage.

For a hybrid sensor network, a computational geometry based approach was proposed for network hole-detection and boundary identification [40]. At first stage, static SRs estimate the size of coverage-hole based on DT construction. Afterwards, mobile SRs are moved to optimal locations to heal the coverage holes and with computational geometry it is determined the required number of extra SRs. The problem of network area hole coverage and ways for recovery through the usage of computational geometry is also examined in [41]. A significant work on methodologies and applications of algorithms to solve this problem appears here. It appears also that it tries to drive attention on computational geometry field suggesting that its inclusion might enhance the solvement of challenges in WSNs. In addition, it is mentioned that SRs detection and communication range, are strongly related with the detection of coverage holes in the network and their recovery. But in all the previously mentioned work, it isn't included the issue of network whole coverage deterioration due to possible obstacles in the ROI and SRs detection problems that might arise from a number of existing TRs.

\section{Problem Statement}

A wide variety of applications and systems exists nowadays which seek for accurate localization. At the same time, a network with a low rate of coverage and a high number of coverage holes, certainly won't operate normally. In [42] Li Q. et al., by designing a reasonable scheme of moving nodes, applied an algorithm which gradually disperse the SRs nodes and improve the coverage effect in the monitoring area. The algorithm used had good performance, reducing the moving distance of nodes resulting in overall network coverage improvement. But, although the results are promising, this work doesn't include the coverage decrease effect of existing TRs in the AOI. As it was showed in [6], network performance and coverage is strictly affected from possible obstacles (TRs) around the SRs of the network. Also, in several existing networks a SRs detecting range has a fixed value. A degradation in QoS is translated in less monitoring and tracking capability by the network of SRs. Thus, for a sufficient level of surveillance each network point should be monitored by a minimum number of $\mathrm{k}$ sensors. The value of $\mathrm{k}$ is a parameter representing the quantity of SRs which are required for a satisfactory level of coverage. A number of $\mathrm{k}$ bigger than one might result in higher QoS [8]. So, in our case and for the procedure of triangulation where a coverage of at least three SRs is needed $(k \geq 3)$, the issue of detection of TRs within a saturated network is a far more complicated issue. Our case of coverage problem can be described as follows: In a two dimensional area A of a certain size we have a set of $\mathrm{SRs} \mathrm{Si}=\mathrm{SR}_{1}, \mathrm{SR}_{2}, \ldots, \mathrm{SRn}$. The size of A might vary as the number of SRs that are used in Si varies as well. Each $\mathrm{SR}$ of $\mathrm{Si}, \mathrm{SRi}$ where $\mathrm{i}=1,2, \ldots, \mathrm{n}$ is located at coordinates (xi, yi) inside $\mathrm{A}$ and has a sensing range of radius $\mathrm{R}$. The SRs of Si have fixed positions, are placed on a grid and monitor around 
in a range $\mathrm{R}$. In area $\mathrm{A}$ a number of TRs also exists which is a set $\mathrm{Tm}=\mathrm{TR}_{1}, \mathrm{TR}_{2}, \ldots, \mathrm{TR}_{\mathrm{k} . .}$ Each $\mathrm{SR}$ of the grid in $\mathrm{A}$ is detecting none or some TRs, but the monitoring area of each SR is blocked by existing TRs and this issue is analyzed further. The network has to find the optimal radius Ropt to apply on the Fixed Sensors Network - FSN, in order to achieve best coverage. Another option that might be used is to activate additional network SRs in order to achieve coverage of at least 3 or more SRs in each sub-region of the network. So, the basic hypotheses that are taken for granted in advance for the network and characterize the way the network acts as an operating system, are the following:

Hypothesis 1. All network SRs of the network have fixed positions.

Hypothesis 2. Each network SR has a 360 degrees field of view and is assumed to detect a TR with

a bearing of $\theta$ with detection error $D_{E R}$,of plusminus $\delta$ degrees.

Hypothesis 3. All the SRs have the same Radius R.

Hypothesis 4. The positions of existing TRs are known. In order to approach this problem and make it more easily understood we give the following definitions:

Definition 1. A location in A is said to be covered by a SRi if it is within the SRi's sensing range location in $\mathrm{A}$ is said to be $\mathrm{j}$-covered if it is within at least $\mathrm{j}$ SRs sensing ranges.

Definition 2. A sub-region Bk in A is a set of points which form a sub-area of A and is covered by at least $\mathrm{k}$ SRs.

Definition 3. A point in any position of the area A can be monitored and a TR can be tracked if its placed at that particular point, if and only if, Definition 1 and 2 are true and that point is not blocked by TRs around it. (A SRi isn't able to detect a TRj beyond a TRi that is detected at a certain bearing $\theta_{\mathrm{i}}$ and by that way it is blocked in that particular bearing $\theta_{\mathrm{i}}$.

\section{Network model}

During various tests, the basic parameter used was the SR bearing (the bearing by which a SR detects a
TR), which is considered to have a detection error plus minus $\mathrm{D}_{\mathrm{ER}}$ for each SR. We assume that each SR has a certain type of detection error. For example, if a SR reports detection of a TR at bearing $340^{\circ}$ and the $D_{\mathrm{ER}}$ for all the network is 3 degrees then the network will use the sector $337^{\circ}$ $343^{0}$ degrees. Thus it will perform data processing taking into account that particular sector, this occurring for each bearing of detection, and for each SR of the FSN. Another parameter is the detection radius $\mathrm{R}$ for each SR. Analyses have been carried out with all SRs having the same value of $\mathrm{R}$, as mentioned in the third Hypothesis. Then, as the network adapts itself to a new state it applies the same optimal Radius, Ropt for each SR. Let us consider as an example two different states, namely State 1 and State2: State $1 S i=S R_{l}, S R_{2}, \ldots, S R n$, same radius R. State $2 \operatorname{Si}($ opt $)=S R_{1}, S R_{2}, \ldots$, $S R n(o p t)$, same radius Ropt. After applying the Ropt the network achieves higher detection levels.

\subsection{Network Grid topology}

For this research there are many grids used for tests. Among them the most commonly used were, the grid of size $1000 \times 1000 \mathrm{~m}$ and a grid of $400 \times$ $400 \mathrm{~m}$. The $\mathrm{m}$ doesn't denote a meter but a unit of length. The first grid includes 121 SRs and various sets of TRs. As shown in Fig.1, 80 TRs imply few areas not covered (in blue). In Fig.2 the number of TRs is increased to 120 , and we can clearly see that the size of the problematic (no coverage) areas is increased, especially in areas which TRs are more closer to each other and more denser. In an area of size $400 \times 400 \mathrm{~m}$ we have a grid of 25 SRs and 50 TRs. The grid of 25 SRs is adequate for testing as increasing the number of the SRs is overloading the system causing delays and this is inappropriate for our research. In Fig.2, the no coverage areas are shown in blue, while the areas with coverage of three or more SRs are brown colored. The SRs are depicted as large stars, whilst the TRs as small stars. By adding a number of extra SRs, we see that the blue (no coverage) area has been significantly reduced Fig.4, compared with Fig.3. In this case the network has become able to detect new TRs. These extra SRs have been added in the areas that needed detection support and extra processing. These SRs are positioned in areas close to dense TRs as their detection beam should not be wide, in order to detect inside those problematic areas. Those SRs groups are inside the yellow colored areas. Each extra SR which is assumed a member of the 
network, transmits the required data to the system and central processing is taking place. With the extra SRs the system is increasing the detection rate thus enhancing performance. It is vital for the network to change its parameters in order to become able to detect new TRs that might enter its area of responsibility.

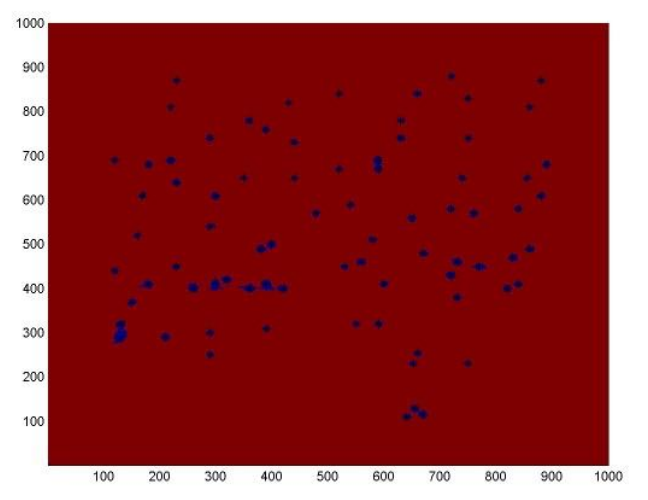

Figure 1. Coverage with $121 \mathrm{SRs}$ and $80 \mathrm{TRs}$

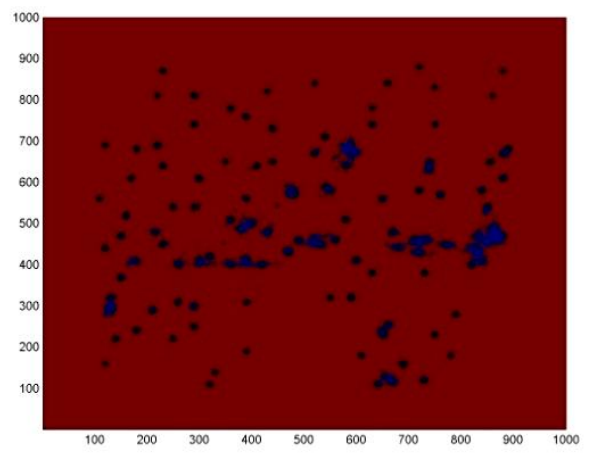

Figure 2. Coverage with $121 \mathrm{SRs}$ and $120 \mathrm{TRs}$ (no coverage is indicated with blue)

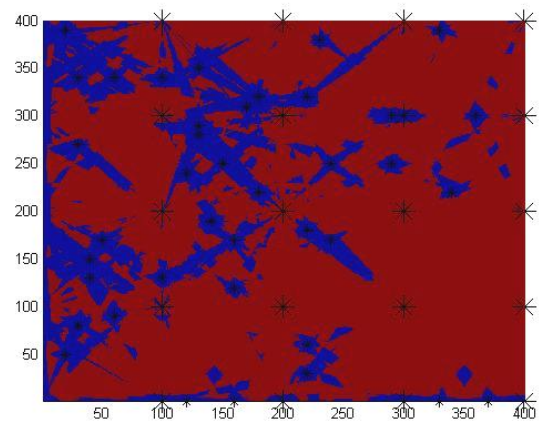

Figure 3. Coverage with $25 \mathrm{SRs}$ and $50 \mathrm{TRs}$

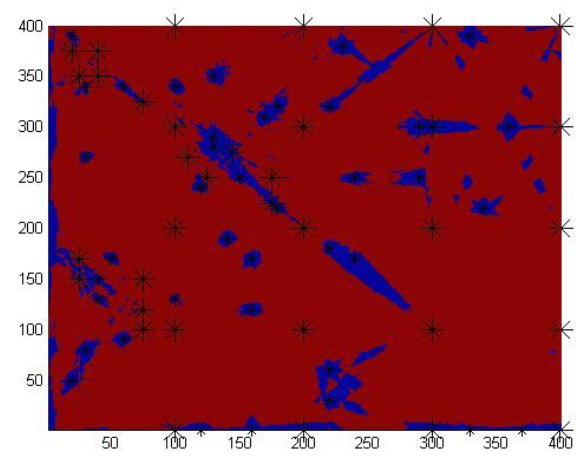

Figure 4. Coverage with $40 \mathrm{SRs}$ and $50 \mathrm{TRs}$ (no coverage is indicated with blue)

\subsection{Network Sensors Blindness}

As it was shown in [43], SRs view is affected by the number of TRs around it. In this paper we will show how SRs blindness has also a significant impact on network detection, leading to network blindness resulting in network coverage performance decrease. A quantification analysis is also presented. We have visualized the gaps in area of coverage that need to be tackled by the network. The software used for the simulations was MatLab version 77 2016b. Knowing the status of the network in advance we were able to apply changes in order to increase its performance. We additionally note that the visualization of coverage gaps is the first step towards the handling of coverage issues. By applying then the correct modifications on the FSN and by adding appropriate groups of SRs, any coverage problems are solved thus keeping the FSN performance in high levels. In the following we also show that a problematic area can be covered by other nearby SRs, by increasing their detection radius or by activating groups of SRs that are located close to this problematic area.

\section{Experiments and discussion}

As it was shown in Fig.4, additional SRs can increase the network performance. The combination of adding extra SRs and changing the detection range $R$ is a method that can give even better results. This method, however, presupposes that the optimal detection radius $\mathrm{R}$ is already known. In order to achieve this result, the FSN gradually increases the $\mathrm{R}$, finally finding the $\mathrm{R}$ that provides 
optimal detection for the whole network. In Fig.4 we can see the value of optimal $\mathrm{R}$ which, in this particular case, is found to be $150 \mathrm{~m}$. The system calculates the level of network coverage by changing gradually the value of $R$. The value of $R$ that gives the best coverage is the optimal $\mathrm{R}$. Processing all these combinations of data requires exhaustive computation. Meaning that the FSN system has to combine each value of SR Radius $\mathrm{R}$ applied to each SR, with the resulting Network coverage. The results produced are forming the figures with curves where we can see the optimal Radius $\mathrm{R}_{\text {opt }}$. In $\mathrm{y}$-axis we can see the relative percentage of area coverage in normalized scale $0-1$. This optimal value of $\mathrm{R}$ is then applied to the FSN and better coverage is achieved. The extra SRs which were activated by the FSN had not been included in the beginning of data processing. These extra SRs are only activated in case extra processing is needed in order to fill gaps in the FSN. This means that the network is then working as a FSN with additional SRs. The new FSN is having a form similar to what shown in Fig.6 (some groups of the 15 SRs are shown). By applying a different test in a grid with 25 SRs and 50 TRs and after adding a group of 15 SRs we can see the difference in coverage performance in Fig.7. We can see that the performance of the network is reached almost hundred percent when we applied the optimal radius. The visualization of the network status allows us to see clearly the areas of non coverage. Then by adding extra groups of SRs and applying the optimal Radius we can further increase performance. In Fig.9 we can see a grid of 25 SRs, including a number of TRs in the area. The SRs can increase the radius of detection $\mathrm{R}$ in order to increase performance. As we can see in Fig.10, in this case each SR has a larger coverage area. This way the network will cover probable problematic areas while, at the same time, a sub-area of the FSN will be covered by more SRs, thus increasing its detection capability.

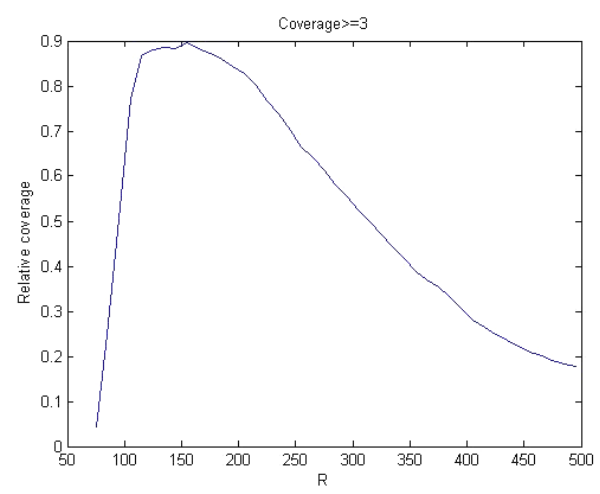

Figure 5. Radius vs Coverage Curve with 25 SRs

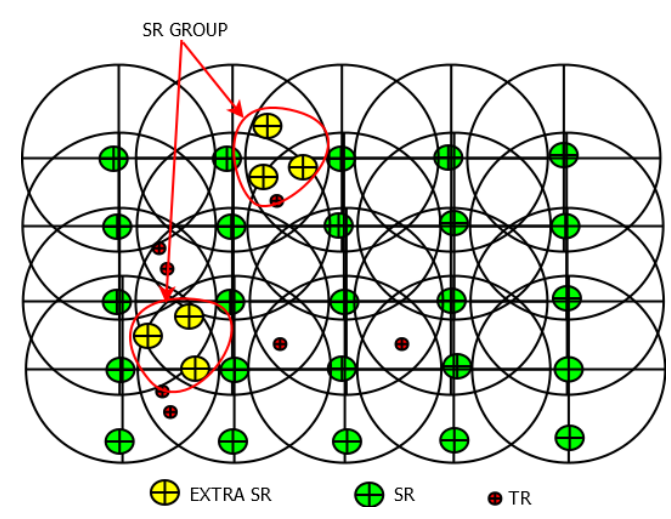

Figure 625 Sensors Grid with Extra Sensors

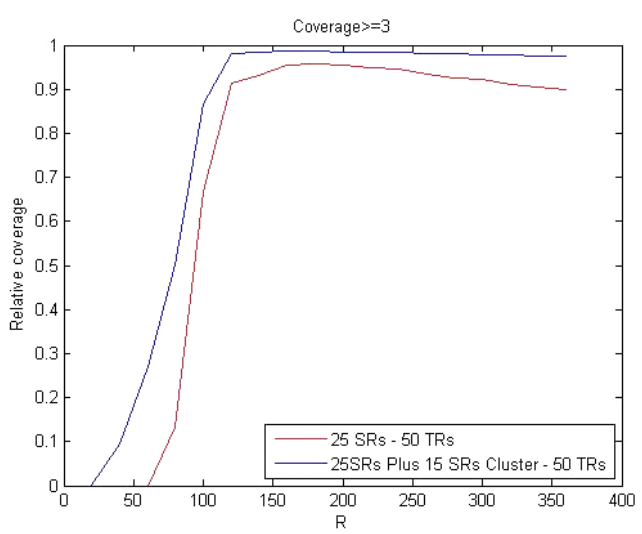

Figure 7. Radius vs Coverage Curve for 25 SRS and 50 TRs with extra sensors

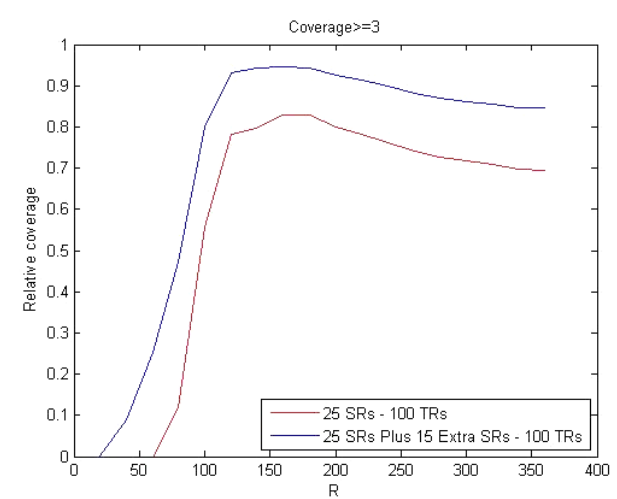

Figure 8. Radius vs Coverage Curve for 25 SRS and 100 TRs with extra sensors 


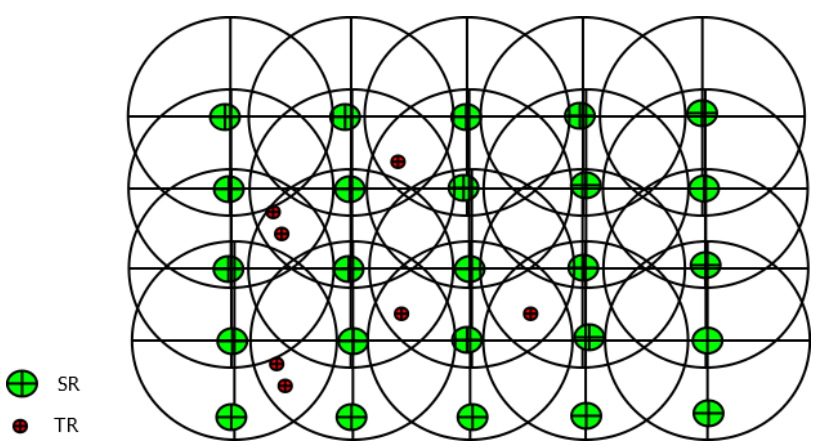

Figure 9. 25 Sensors Grid with radius R and TRs

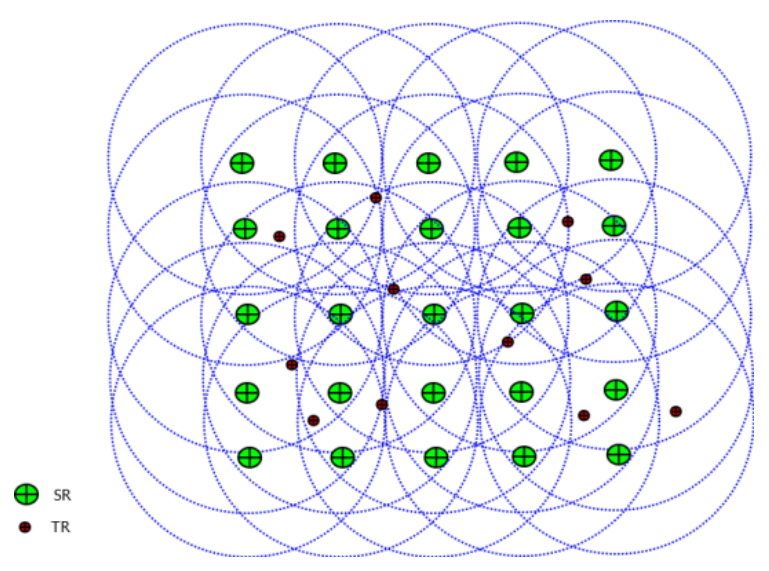

Figure 10. 25 Sensors Grid with increased radius $R$

\subsection{Network Detection Performance}

Network detection is affected by a number of factors that might alter its accuracy and its performance. As the network has to operate as a system, the user has to be aware in advance for any possible changes. As it was shown before, as the number of TRs is increased in the area, the system has to apply changes in order to keep a high detection rate. In the following Fig. 11 we have a network grid of 25 SRs. We will show how detection performance is lowered due to TRs blockage in the area. In Fig.12, we have a network grid of 25 SRs and 12 TRs. Here, we can see the sectors of detection beams assuming that we have an error of plus-minus 2 degrees. As we see in Fig.12, SRs 1, 2, 3, 4, 6, 7, 8, $9 \ldots, 11$ till 14 and 16 are having beams detecting TRs. In this case the network detection rate can be measured with the formula (1) which was presented in [5]. The existing bearings and the existing triangulations, ETRNs are the basic parameters that affect the detection rate.

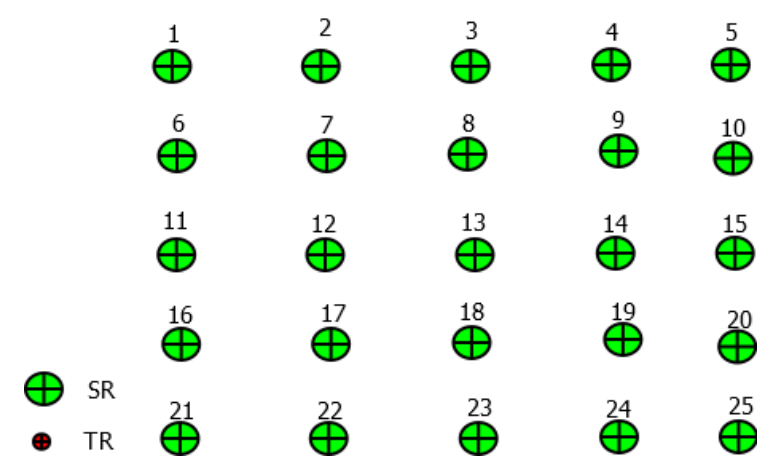

Figure 11. 25 Sensors Grid

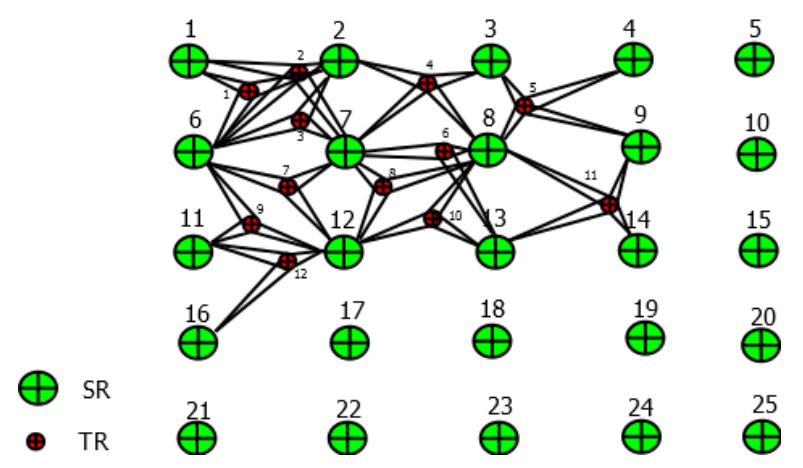

Figure 12. 25 Sensors Grid with TRs and plus-minus beam detection error

NDrate $=100-\left[\left(S R_{D F}+N B_{B L}(2-k)\right)+E T R N s\right] / 3$

In case that we have more than 100 SRs the formula (1) takes the form of (2)

$N$ Drate $=\mathrm{n}-\left[\left(S R_{D F}+N B_{B L}(2-k)\right)+E T R N s\right] / 3$

$\mathrm{SR}_{\mathrm{DF}}$ is defined as the number of SRs which will fail to detect TRs for any reason. NBL is given by formula (3) and it is defined as the sum of blindness of each SR divided with the number of SRs, $n$.

Quotient $\mathrm{k}$ is defined as,

$k=1 /$ ETRNs, where: $0<\mathrm{k}<1$.

In this particular case, we assume that the network has a zero failure of SRs detection (all the SRs are working properly), so $S R_{D F}=0$. Also, in our case,

$$
\text { ETRNs }=12, \text { so } k=1 / 12=0.083
$$


the number of existing triangulations ETRNs is 12 ,we have to calculate also the network blindness NBL. We can calculate it with the following formula (3) which was presented in [6].

$$
N_{B L}=\left(B 1+B 2+B 3+\ldots+B_{n}\right) / n
$$

Where $B_{1}+B_{2}+B_{3}+\ldots+B_{n}$ is the sum of blindness of each SR and $n$ is the number of SRs with bearings. Blindness (4) for each SR was defined in [6].

$$
S R_{B L}=R_{S R s} \times 2 * S R_{E R} / 360 * 100 \%
$$

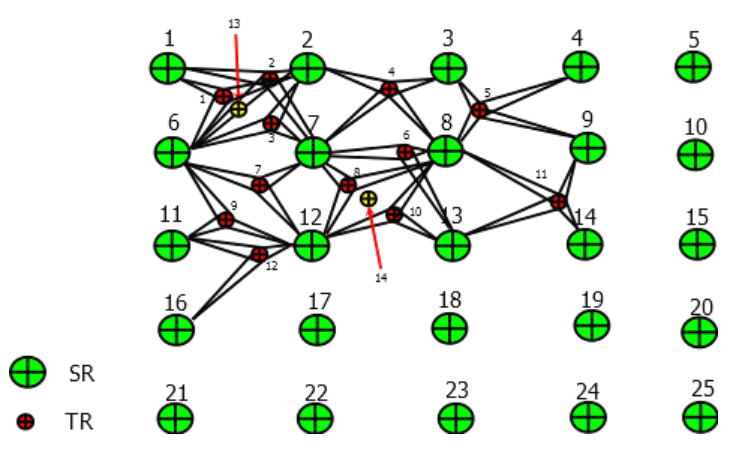

Figure 13. 25 Sensors Grid with TRs detection beams and extra TRs

Where $R_{S R s}$ is the number of radials per $S R$ and $\mathrm{SR}_{\mathrm{ER}}$ is the common bearing error for each SR of the network. The number $360^{\circ}$ is related with the 360 degrees coverage for a SR. With formula (4) we define each SR's blindness as a percentage. We assume that our network has a network error of plus-minus 2 degrees. We have a grid of $25 \mathrm{SRs}$, but not all of them will detect TRs. The SRs that have bearings sectors are:

$S R_{1}, \ldots, S R_{2}, S R_{6}, \ldots, S R_{9}, S R_{11}, \ldots, S R_{14}$ and $S R_{16}$

a total of 13 SRs. Hence, $n=13$.

The FSN system has to calculate overall blindness using data from all sensors. After applying the formula (3) for each SR we can find the network blindness

$$
\begin{gathered}
N B_{B L}=\left(B_{1}+B_{2}+B_{3}+B_{4}+B_{6}+B_{7}+B_{8}+B_{9}+B_{11}\right. \\
\left.+\ldots B_{12}+B_{13}+B_{14} \quad \ldots+B_{16}\right) / n, \\
N B_{B L}=39.96 / 13=3.07
\end{gathered}
$$

Hence,

$$
\begin{gathered}
\text { NDrate }=100-\left[\left(\operatorname{SR}_{D F}+N B_{B L}(2-k)\right)+\text { ETRNs }\right] / 3 \\
\text { NDrate }=100-[(0+3 \times(2-0.083))+12] / 3 \\
\text { NDrate }=100-5.917=94.083
\end{gathered}
$$

That means that the detection rate of our network in the current state, is 94.083 . This number is going to be further decreased when new TRs will enter the area and when some SRs fail to detect TRs. In this case $S R_{D F}$ will also be increased. In Fig.13 we have added 2 more TRs resulting in 14 TRs. Here, we can see the sectors of detection beams assuming that we have an error of plus-minus 2 degrees. Since these two TRs are blocked by other TRs, we need to detect them using other SRs of the grid. SRs 1,6,7 are blocked by other TRs while there is a low probability that $\mathrm{SR}_{2}$ will detect $\mathrm{TR}_{13}$. The same problem appears to happen on $\mathrm{TR}_{14}$ where $\mathrm{SRs} 7$ and 13 are blocked. In this case the network has to change its parameters and apply different radius for some SRs of the network. Otherwise we won't be able to apply the triangulation procedure and detect them.

\subsection{Network performance with Bearing detection error}

As previously mentioned, one of the fundamental characteristics that affect the network detection performance is the error of bearing detection. In the following Fig.14, $a$ and b, we have four different curves of bearing errors at 2, 4, 6 and $8^{0}$ degrees. The Fig.14 resulted from a grid of 25SRs and 10TRs, whilst Fig. 15 resulted from a grid of 25SRs and 30TRs and Fig. 16 resulted from a grid of 25SRs and 50TRs So, we draw the conclusion that, as the beam error is gradually increased, the coverage is reduced. In addition, by adding a number of extra TRs, $(10$ to 50$)$ we see that the quality of coverage is significantly reduced. 


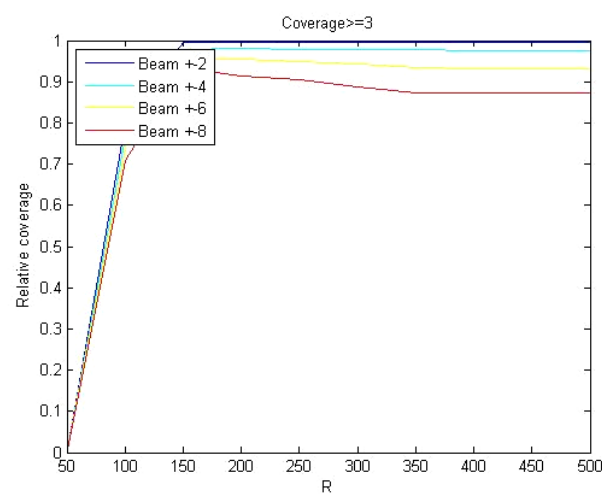

Figure 14. Coverage Curves with different beam errors with 25 SRs and 10 TRs

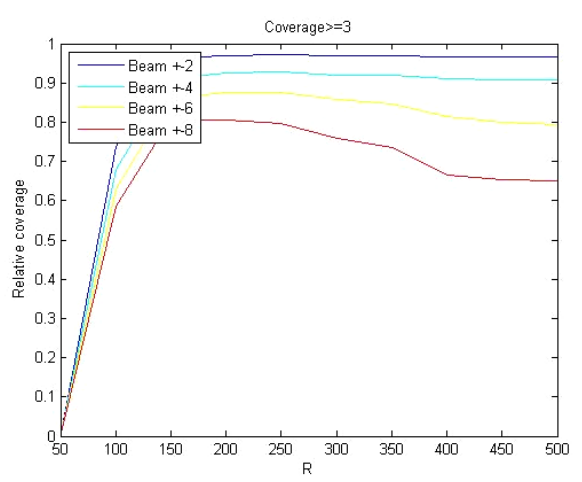

Figure 15. Coverage Curves with different beam errors with $25 \mathrm{SRs}$ and $30 \mathrm{TRs}$

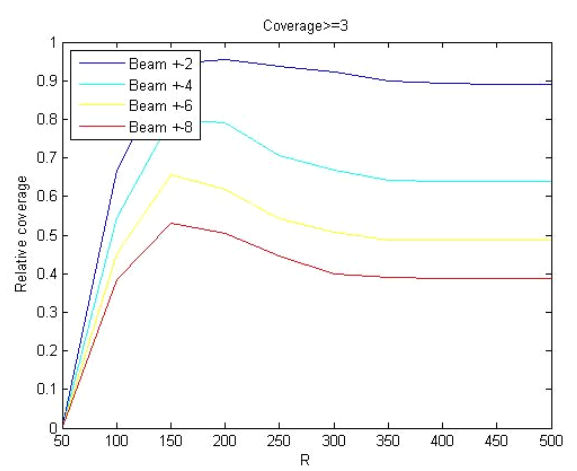

Figure 16. Coverage Curves with different beam errors with $25 \mathrm{SRs}$ and $50 \mathrm{TRs}$

Also, the size of no coverage areas is gradually increased as the beam error is increased as well. In Fig. 17 - Fig.20, we see this gradual change when we change from 2 to 4,6 and finally 8 degrees It is obvious that the FSN is becoming blind and detection becomes impossible in its interior, when beam error is high, Fig.19. With this visualization tool we can easily control the effect of beam error in the network and its coverage performance. Mostly, beam error of maximum 4 degrees is used for the processing of data as a great amount of error is affecting also the triangulation s resulting in a high number of false triangulation s, an additional problem which has to be avoided.

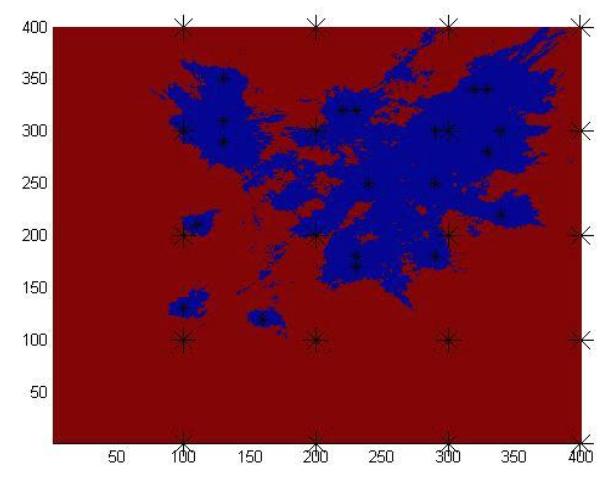

Figure 17. Coverage with beam error of 2 degrees (no coverage is indicated with blue)

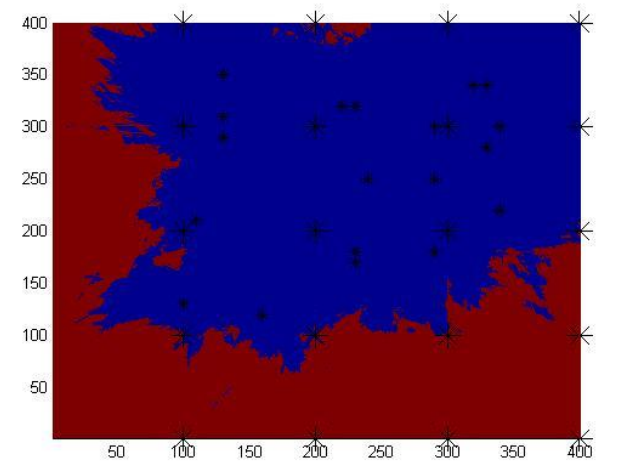

Figure 18. Coverage with beam error of 4 degrees (no coverage is indicated with blue)

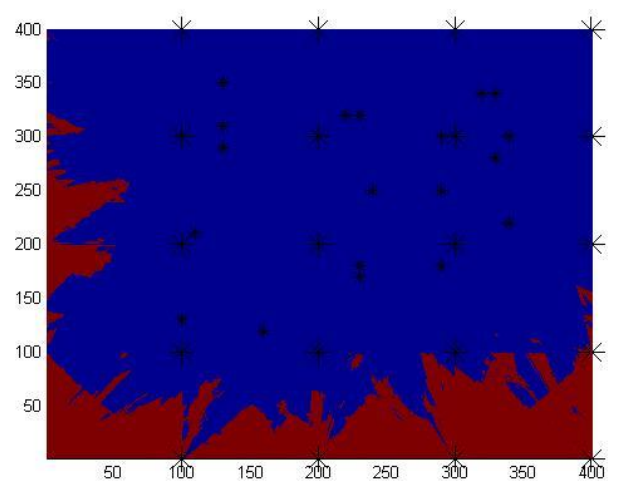

Figure 19. Coverage with beam error of 6 degrees (no coverage is indicated with blue) 


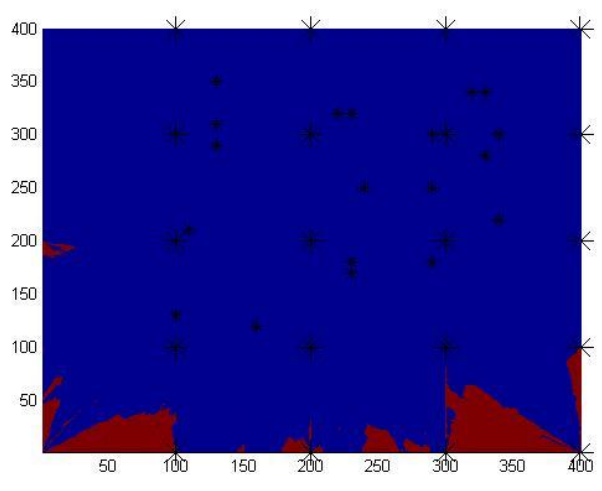

Figure 20. Coverage with beam error of 8 degrees (no coverage is indicated with blue)

Also, and as we see in Fig.21 where the coverageRadius curve for $8^{0}$ degrees error is shown, we see that we have a sharp decrease when the Radius is increased beyond $130 \mathrm{~m}$. That means that the network becomes almost blind beyond that limit of $\mathrm{R}$. This information is significant as we can apply the required changes to the system, enabling us to keep its performance high. In this case as it was mentioned earlier, we use the same radius $\mathrm{R}$ for each SR. In the next section there are a number of tests which were executed in order to present the network changes with varying topologies and different SRs number combined with varying radius of three values.



Figure 21. Radius vs Coverage plot with 25 SRs, 50 TRs and $8^{0}$ degrees beam error

\subsection{Network performance with different sensor radius.}

In Fig.22, we can see coverage with different fixed radiuses (Small- S, Medium M and Large L) modes in a grid with 25 SRs. Here, with different tests we will show how network coverage is affected when all the SRs have a fixed radius $\mathrm{R}$ with increasing number of TRs in the area. We will apply this mode in a network grid with 6 SRs, as in Fig.23 after adding 10, 20, 30 TRs. We use for radius a values range of $450 \mathrm{~m}$ and then we split the $450 \mathrm{~m}$ radius range to three values of S-M-L, Small $\mathrm{S}$ 150m,Medium M 300m and Large L, 450m.



Figure 22. 25 Sensors Grid with S-M-L Radius mode

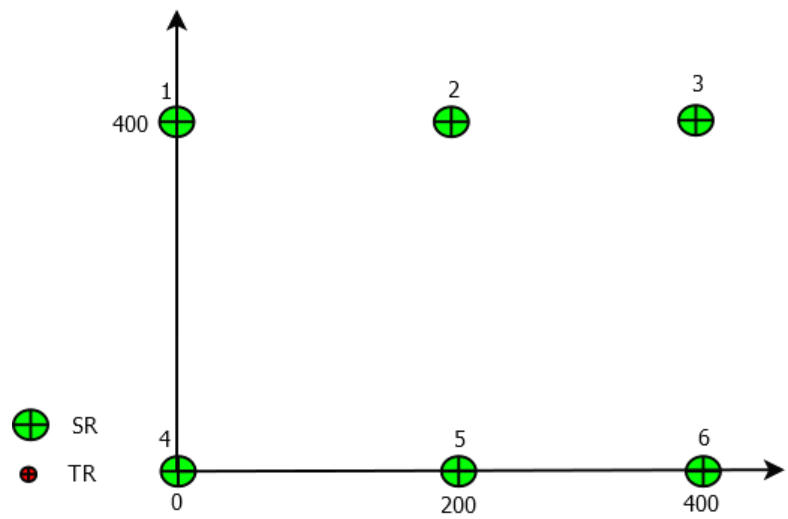

Figure 23. 6 Sensors Grid with S-M-L Radius mode

\section{TEST 1}

Number of Sensors $=6$

Number of TRs $=10$

Number of Radius Variations $=3$ (S-M-L)

Optimal Relative Coverage $>=3=0.855656$

With the Radius Combination $=$ L L L L L L

with the Radius Combination $=$ M M M M M M we

had: Relative Coverage $(>=3)=0.700519$ 
and with the Radius Combination $=$ S S S S S S we had: Relative Coverage $(>=3)=0.239200$.

\section{TEST 2}

Number of Sensors $=6$

Number of TRs $=20$

Number of Radius Variations $=3(\mathrm{~S}, \mathrm{M}, \mathrm{L})$

The results are: Optimal Relative Coverage $(>=3)$ $=0.653656$

With Optimal Radius Combination $=$ L L L L L L, with the Radius Combination $=$ M M M M M M we had: Relative Coverage $(>=3)=0.449987$

with the Radius Combination $=$ S S S S S S we had: Relative Coverage $(>=3)=0.167350$.

Fig.24 shows the network coverage with radius R, Large - $\mathrm{L}$ which is the optimal value of 0.855656 for the network.Fig. 25 shows the network coverage with radius $R$, in $L$ mode. We see clearly that the area of 3 SRs coverage is decreased significantly compared with Fig.19. After increasing the number of TRs to 30 we had the following results:

\section{TEST 3}

Number of Sensors $=6$

Number of TRs $=30$

Number of Radius Variations $=3(\mathrm{~S}-\mathrm{M}-\mathrm{L})$

Optimal Relative Coverage $(>=3)=0.470994$

Optimal Radius Combination $=$ L L L L L L

With the Radius Combination $=$ M M M M M M we had: Relative Coverage $(>=3)=0.317294$

with the Radius Combination $=$ S S S S S S we had: Relative Coverage $(>=3)=0.127200$.

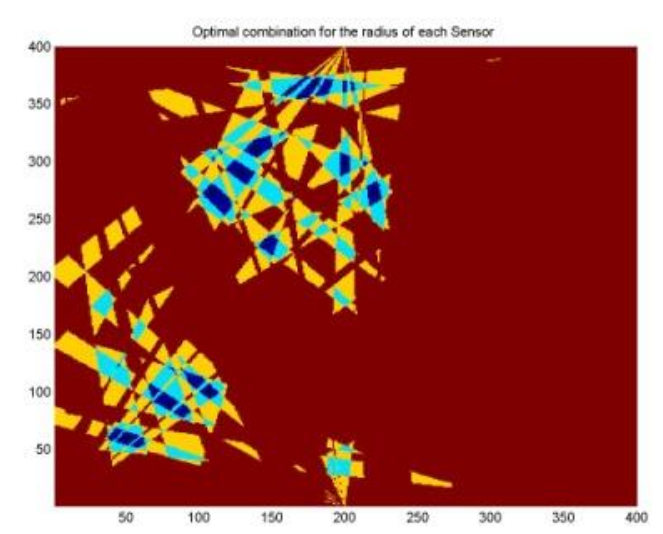

Figure 24. 6 Sensors- Grid - 10 TRs, S-M-L Radius mode - Colours definition Zero coverage, Light blue, Coverage from one SR, Yellow, Coverage from

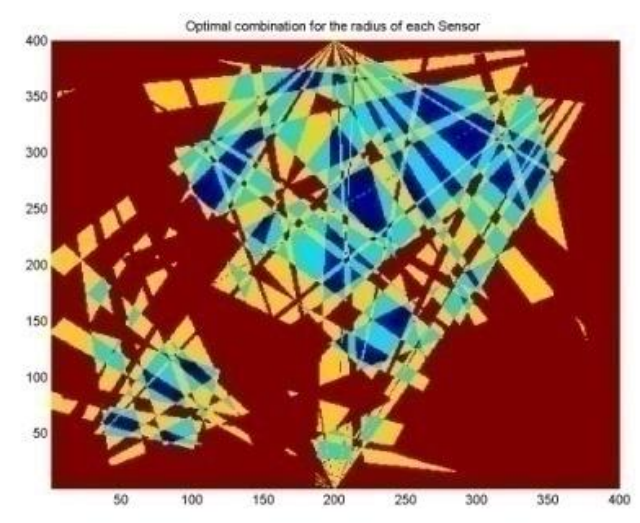

Figure 25. 6 Sensors- Grid - 20 TRs, S-M-L Radius mode - Colours definition Zero coverage, Light blue, Coverage from one SR, Yellow, Coverage from two SRs, Red, Coverage from three or more SRs.

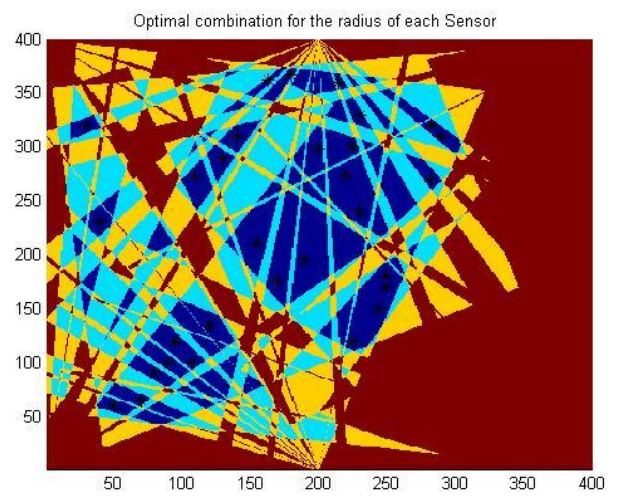

Figure 26. 6 Sensors- Grid -30 TRs, S-M-L Radius mode Colours definition same as previous figures.

In Fig.26 we have the visualization of network coverage with these parameters in L mode and we see that the covered area is reduced significantly (blue colour) and more SRs are needed in order to tackle the network saturation problem.

\subsubsection{Network performance with three sensor detection radius (Small(S), Medium(M), Large(L))}

As every change in $\mathrm{R}$ is affecting each SRs coverage resulting in network coverage change, we should note that a fixed radius $\mathrm{R}$, might not be the best option, when optimal coverage is to be achieved. For that reason we decided to examine and test the option of a mode for detection with each 
sensor having one of three fixed radiuses, resulting in different radiuses at the same time (S-M-L).That way the Hypothesis 3, "All the SRs have the same Radius R" is changed to "Each SR of the system might have a varying radius $\mathrm{R}, \mathrm{S}-\mathrm{M}-\mathrm{L}$ ". Here, after executing some tests we will show that a different combination of SRs radius $\mathrm{R}$ gives us the best coverage. In the previous tests from one to three we saw that the system has the optimal coverage when increasing the radius of the SRs and the system applies large $L$ radius for the whole network. In this case we will show that the system might use a varying radius $\mathrm{R}$ applied on each $\mathrm{SR}$ and this combination is the optimal key for the network. The varying radius $R$ for each SR can take three values S-M-L. In these tests, we used two network grid topologies, one with $10 \mathrm{SRs}$ in a $400 \mathrm{x} 400$ marea and one with 15 SRs spread in a same area which is depicted in Fig.27 and Fig.28 respectively.

\section{TEST 4}

Number of Sensors $=10$

Number of Transmitters $=10$

Number of Radius Variations $=3$

Optimal Radius Combination= LSL LM M M L L L Optimal Relative Coverage $(>=3)=0.961219$

All combinations were tested to find the optimal relative coverage for the network. This exhaustive search approach usually takes days to find the optimal combination with high number of SRs and TRs.

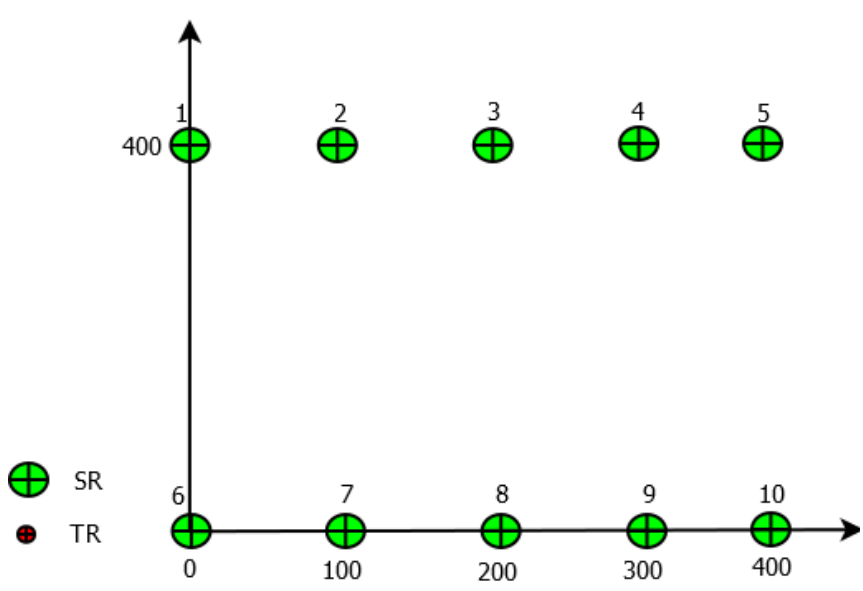

Figure 27. 10 Sensors Grid with S-M-L Radius mode
However, optimization techniques can be employed to minimize the run time.

\section{TEST 5}

Number of Sensors $=10$

Number of Transmitters $=20$

Number of Radius Variations $=3$

Optimal Radius Combination=LS M L LMM M L L

Optimal Relative Coverage $(>=3)=0.869519$

\section{TEST 6}

Number of Sensors $=10$

Number of Transmitters $=30$

Number of Radius Variations $=3$

Optimal Radius Combination $=$ L S S LMMM S L L

Optimal Relative Coverage $(>=3)=0.753813$

In the previous tests 4,5,6 we saw that every time we added 10 TRs the Optimal Relative Coverage was lowered by 10 percent. In the next test 7 we will show that by adding only 5 more SRs the system rises its performance at a high level even with 30 TRs. In the next TEST 7 we increase the number of TRs to 30 in order to examine the affection of this change in network performance.

\section{TEST 7}

Number of Sensors $=15$

Number of Transmitters $=30$

Number of Radius Variations $=3$

Optimal Radius Combination $=$ S M S M S S S S S

S S S S S S

Optimal Relative Coverage $(>=3)=0.928988$

In the previous tests it was shown that the system can decrease the radius of some SRs from $L$ to $M$ or $\mathrm{S}$ in order to tackle the saturation and find the optimal key of SRs radius which gives us the optimal network coverage. In this case with 15 SRs the number of permutations to be executed by the system is really high and it took several days of running in order to receive the results. But the difference of coverage compared with the previous grid of 10 SRs is great and then we achieved a high level of coverage ( 92 percent) after increasing the SRs from ten to fifteen and applying the optimal SRs. 


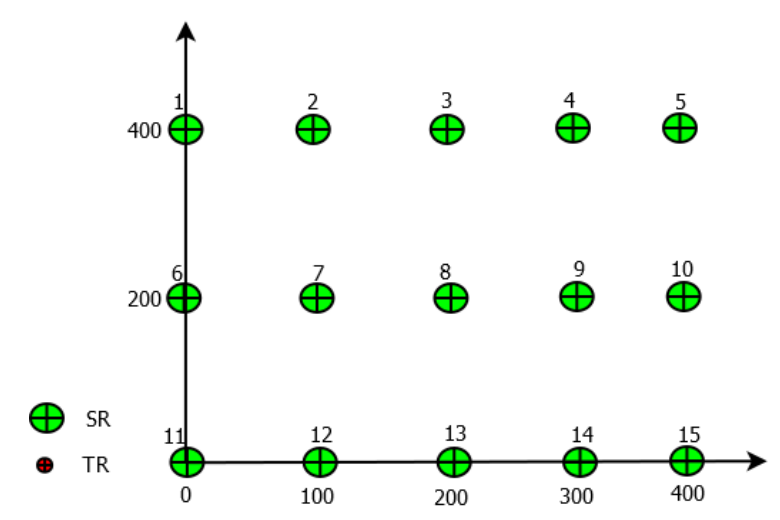

Figure 28. 15 Sensors Grid -S-M-L Mode

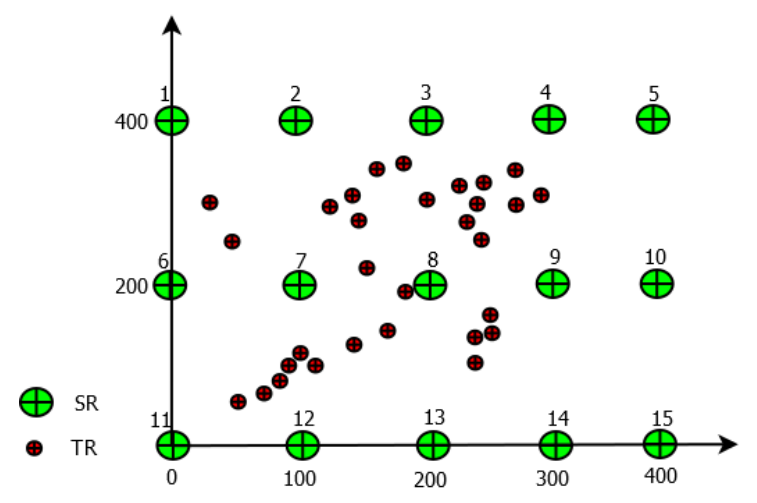

Figure 29. 15 Sensors Grid 30 TRs - S-M-L Mode

In the next chart Fig.30 we have a depiction of how coverage is affected when more TRs enter the area in each grid. We see that for the grid of 6 SRs, a ten TRs increase, results a coverage decrease of twenty percent. While for the grid of ten SRs, after adding ten TRs, the decrease reaches ten percent. As far as the grid of 15 SRs coverage percentage is concerned, only the value related with 30 TRs is shown. The case where different $\mathrm{R}$ is to be applied for each SR remains to be examined thoroughly, Fig.31.

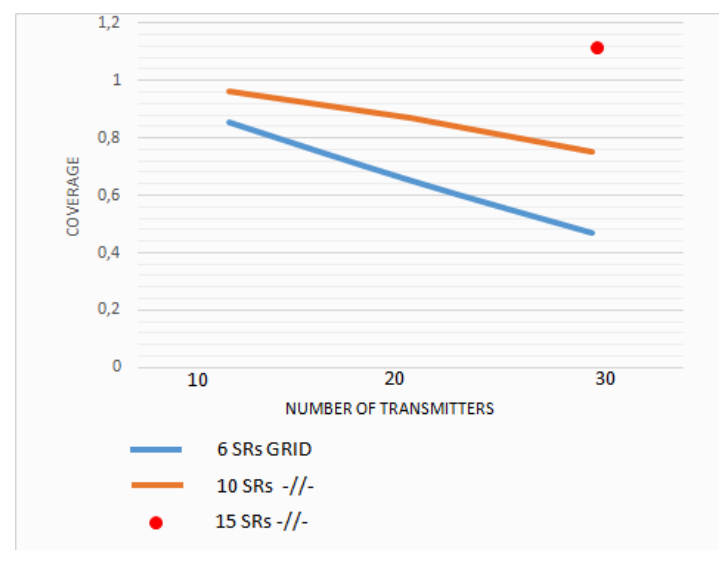

Figure 30. Transmitters-Coverage Chart with varying Grids radius key

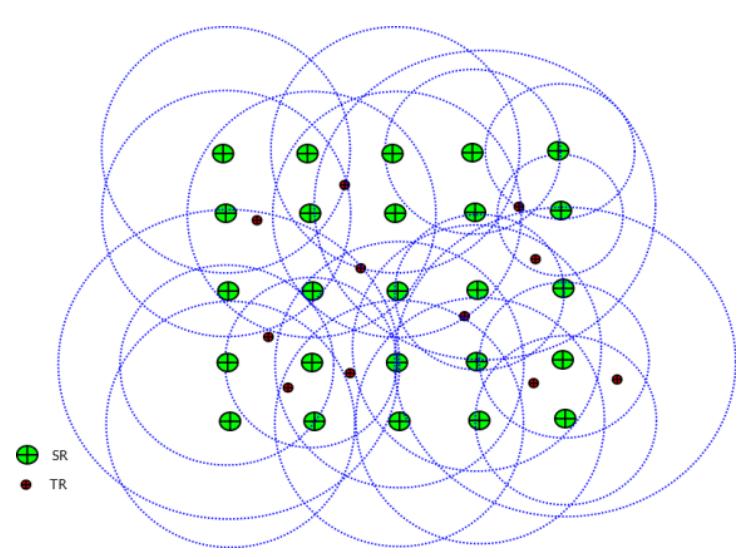

Figure 31. 26 Sensors Grid with varying Radius

\section{Contributions}

The contributions of this work is the development of a strategy to evaluate the performance of this particular type of FSN, based on the problem statement. Network Area Coverage Holes are identified and areas of non coverage of three or more SRs for triangulation are visualized for a system user. By that way we have an evaluation of network status and its problematic areas in any state and with a certain number of existing TRs.

1.Whereas most previous works focused on considering a sub-area or a network hole [20], $[25],[28]$ etc, in this work the whole FSN AOI is 
considered but this time by taking into consideration the existence of previous TRs which operate as obstacles in the area and affect the FSN coverage. This is far more different from previous works which mainly focus on coverage of region or subregion by a single SR and not by three minimum SRs $(k \geq 3)$ which will perform localization with triangulation of a new TR.

2.Furthermore, instead of trying to cover an area by adding more SRs in a sub-area of the FSN the system applies optimization of coverage by two different ways. Firstly, by finding and applying an optimal radius $\mathrm{R}$ same for each SR and secondly by finding and applying an optimal combination of different R for each SR which we name "key".

3. In earlier works [33], [38], [41], authors are seeking for coverage holes and coverage healing by adding a SR or some SRs that might cover an area or a sub-area in the Region of Interest (ROI). This is different from our work in which the FSN is selfaware of its state continuously, and it can apply changes from state to state in order to achieve a high level of coverage in a region by a minimum number of SRs and $\mathrm{k} \geq 3$.

4.Also, in this work new knowledge is added to existing knowledge, which is the following:

a. It is presented the new concept of FSN blindness $\mathrm{N}_{\mathrm{BL}}$ for a FSN for localization with triangulation with a certain fixed error for each $\mathrm{SR}_{\mathrm{er}}$ in an area with existing TRs.

b It is presented the new concept of Network detection rate $\mathrm{ND}_{\text {rate }}$ for that particular type of FSN.

\section{Conclusions}

This research incorporates substantial work for a FSN system for localization via triangulation able to detect TRs which enter an Area of Interest. It was shown that as the number of TRs increase in the area issues of saturation and SRs blindness appear in the network. The issue of saturation and blindness in the network is approached and analyzed thoroughly and it is proved that the network has to apply adequate optimization methods in order to tackle these problem. Two method of optimization for this type of network are presented which are the optimal radius $\mathrm{R}$ and the optimal Key radius $\mathrm{R}$ of Small Medium Large S-M-L where a combination of different radius $\mathrm{R}$ for each SR is applied in the FSN in order to achieve the optimal coverage resulting in overall higher network detection performance. These issues of blindness, network saturation and optimal detection performance for this type of FSN for detection with triangulation hasn't been researched up to now and it forms a new contribution to existing knowledge.

Author Contributions: Conceptualization, M.S.; Methodology, Simulation and Optimization M.S. and M.Z..; writing - original draft preparation, E.V.; writing - review and editing, M.S. and M.Z.; visualization, M.S. and E.V.; supervision, E.A. and N.R.; All authors have read and agreed to the published version of the manuscript.

Funding: This research received no external funding.

\section{Acknowledgments:}

I would like to express my gratitude to Professor Rajagopal and Professor Antonidakis, my research supervisors, for their patient guidance, and useful critiques of this research work. I would also like to thank Professor Zakinthinaki for her valuable contribution in producing the adequate Matlab software for the tests of this research.

Conflicts of Interest: The authors declare no conflict of interest.

\section{Abbreviations}

The following abbreviations are used in this manuscript:

FSN Fixed Sensors Network

SRs Sensors

TRs Transmitters

ETRNs Existing Triangulations

QoS Quality of Service

\section{References}

[1] G. Fan and S. 363 Jin, Coverage problem in wireless sensor network: A survey, J. Netw., vol. 5, no. 9 , pp. 364 1033-1040, 2010.

[2] Nema, S, Shukla, N.: A review on coverage factors in wireless sensor networks. Int. J. Adv. Res. Comput. Eng. Technol. (IJARCET) 2(12), 1-5 (2013.). 
[3] Sohrabi, K., Gao, J., Ailawadhi, V., \& Pottie, G. J. (2000). Protocols for self-organization of a wireless sensor network. IEEE personal communications, 7(5), 16-27.

[4] D. Dardari, P. Closas, and P. M. Djuri c, Indoor tracking: Theory, methods, and technologies, IEEE Transactions on Vehicular Technology, vol. 64, no. 4, pp. 1263-1278, Apr. 2015.

[5] Y. Gu, A. Lo, and I. Niemegeers, A survey of indoor positioning systems for wireless personal networks, IEEE Communications surveys and tutorials, vol. 11, no. 1, pp. 13-32, 2009.

[6] A. R. Kulaib, R. M. Shubair, M. A. Al-Qutayri, and J. W. P. Ng, "An overview of localization techniques for wireless sensor networks," in Proc. Int. Conf. Innov. Inf. Technol. (IIT), Apr. 25-27, 2011, pp. 167-172.

[7] Zhu, C., Zheng, C., Shu, L., Han, G. (2012). A survey on coverage and connectivity issues in wireless sensor networks. Journal of Network and Computer Applications, 35(2), 619-632.

[8] Coluccia, Angelo, \& Fascista, Alessio. (2019). A Review of Advanced Localization Techniques for Crowdsensing Wireless Sensor Networks. Sensors (Basel, Switzerland), 19(5), 988.

[9] Maheshwari, Aastha, \& Chand, Narottam. (2018). A Survey on Wireless Sensor Networks Coverage Problems. In Proceedings of 2nd International Conference on Communication, Computing and Networking (Vol. 46, Lecture Notes in Networks and Systems, pp. 153-164). Singapore: Springer Singapore.

[10] Khelifi, F., Bradai, A., Benslimane, A., Rawat, P., \& Atri, M. (2019). A survey of localization systems in internet of things. Mobile Networks and Applications, 24(3), 761-785.

[11] Chai, Senchun, Wang, Zhaoyang, Zhang, Baihai, Cui, Lingguo, \& Chai, Runqi. (2020). Localization Technology for Wireless Sensor Networks. In Wireless Sensor Networks (Wireless Networks, pp. 69-141). Singapore: Springer Singapore.

[12] Bin Waheed, M. Hamza, Rais, Rao Naveed Bin, Khan, Hassan, Bano, Mukhtiar, \& Gilani, Syed
Sherjeel A. (2020). Indoor Mobile Localization Using Wireless Sensor Networks (WSNs). In Web, Artificial Intelligence and Network Applications (Vol. 1150, Advances in Intelligent Systems and Computing, pp. 341-351). Cham: Springer International Publishing.

[13] https://en.wikipedia.org/wiki/Triangulation.

[14] Anurag, A., Priyadarshi, R., Goel, A., \& Gupta, B. (2020, February). 2-D coverage optimization in WSN using a novel variant of particle swarm optimisation. In 2020 7th International Conference on Signal Processing and Integrated Networks (SPIN) (pp. 663-668). IEEE.

[15] Singh, S., Kumar, S., Nayyar, A., Al-Turjman, F., \& Mostarda, L. (2020). Proficient QoS-Based Target Coverage Problem in Wireless Sensor Networks. IEEE Access, 8, 74315-74325.

[16] Hanh, N. T., Binh, H. T. T., Hoai, N. X., \& Palaniswami, M. S. (2019). An efficient genetic algorithm for maximizing area coverage in wireless sensor networks. Information Sciences, 488, 58-75.

[17] Katti, A. (2019). Target coverage in random wireless sensor networks using cover sets. Journal of King Saud University. Computer and Information Sciences, Journal of King Saud University. Computer and information sciences, 2019-05.

[18] Saadi, N., Bounceur, A., Euler, R., Lounis, M., Bezoui, M., Kerkar, M., \& Pottier, B. (2020). Maximum lifetime target coverage in wireless sensor networks. Wireless Personal Communications, 111(3), 1525-1543.

[19] Si, Pengju, Ma, Junlei, Tao, Fazhan, Fu, Zhumu, \& Shu, Lei. (2020). Energy-Efficient Barrier Coverage With Probabilistic Sensors in Wireless Sensor Networks. IEEE Sensors Journal, 20(10), 5624-5633.

[20] Ammari, H. M. (2019). Investigating physical security in stealthy lattice wireless sensor networks using k-barrier coverage. Ad Hoc Networks, 89, 142-160. 
[21] Rani, J., \& Anand, V. (2020). The Coverage Issues in WSNs. Asian Research Journal of Mathematics, 1-10.

[22] Si, Pengju, Ma, Junlei, Tao, Fazhan, Fu, Zhumu, \& Shu, Lei. (2020). Energy-Efficient Barrier Coverage With Probabilistic Sensors in Wireless Sensor Networks. IEEE Sensors Journal, 20(10), 5624-5633.

[23] Das, S., \& Debbarma, M. K. (2019). A survey on coverage problems in wireless sensor network based on monitored region. In Advances in Data and Information Sciences (pp. 349-359). Springer, Singapore.

[24] Wang, Z., Cao, Q., Qi, H., Chen, H., \& Wang, Q. (2017). Cost-effective barrier coverage formation in heterogeneous wireless sensor networks. Ad Hoc Networks, 64, 65-79.

[25] Al-Karaki JN, Gawanmeh A (2017) The optimal deployment, coverage, and connectivity problems in wireless sensor networks:revisited. IEEE Access 5:18051-18065.

[26] Wang, Y., Wu, S., Chen, Z., Gao, X., \& Chen, G. (2017). Coverage problem with uncertain properties in wireless sensor networks: A survey. Computer Networks, 123, 200-232.

[27] Ababneh, A. A. (2019). Target localization accuracy improvement via sensor mobility. International Journal of Parallel, Emergent and Distributed Systems, 34(5), 594-614.

[28] Kandris, D., Nakas, C., Vomvas, D., \& Koulouras, G. (2020). Applications of wireless sensor networks: an up-to-date survey. Applied System Innovation, 3(1), 14.

[29] Ren, K., Karlsson, J., Liuska, M., Hartikainen, M., Hansen, I., \& Jørgensen, G. H. (2020). A sensor-fusion-system for tracking sheep location and behaviour. International Journal of Distributed Sensor Networks, 16(5), 1550147720921776.

[30] Yu, J., Liang, D., Gong, X., \& Song, X. (2018). Impact localization for composite plate based on detrended fluctuation analysis and centroid localization algorithm using FBG sensors. Optik, 167, 25-36.

[31] Tarnaris, K., Preka, I., Kandris, D., \& Alexandridis, A. (2020). Coverage and k-Coverage Optimization in Wireless Sensor Networks Using Computational Intelligence Methods: A Comparative Study. Electronics, 9(4), 675.

[32] Li, Y., Qi, G., \& Sheng, A. (2019). Optimal deployment of vehicles with circular formation for bearings-only multi-target localization. Automatica, 105, 347-355.

[33] Wu, M. (2020, February). An Efficient hole Recovery Method in Wireless Sensor Networks. In 2020 22nd International Conference on Advanced Communication Technology (ICACT) (pp. 530-535). IEEE.

[34] Zhai, S., Tang, Z., Wang, D., Li, Q., Li, Z., Chen, X., ... \& Wang, Z. (2018). Enhancing received signal strength-based localization through coverage hole detection and recovery. Sensors, 18(7), 2075.

[35] Hajjej, F., Hamdi, M., Ejbali, R., \& Zaied, M. (2020). A distributed coverage hole recovery approach based on reinforcement learning for Wireless Sensor Networks. Ad Hoc Networks, 101, 102082 .

[36] Singh, P., \& Chen, Y. C. (2020). Sensing coverage hole identification and coverage hole healing methods for wireless sensor networks. Wireless Networks, 26(3), 2223-2239.

[37] So-In, C., Nguyen, T. G., \& Nguyen, N. G. (2019). An efficient coverage hole-healing algorithm for area-coverage improvements in mobile sensor networks. Peer-to-Peer Networking and Applications, 12(3), 541-552.

[38] Zhang, G., Qi, C., Zhang, W., Ren, J., \& Wang, L. (2017). Estimation and healing of coverage hole in hybrid sensor networks: A simulation approach. Sustainability, 9(10), 1733. 
[39] Gupta, S. K., Kuila, P., \& Jana, P. K. (2016). Genetic algorithm approach for k-coverage and $\mathrm{m}$ connected node placement in target based wireless sensor networks. Computers \& Electrical Engineering, 56, 544-556.

[40] Baha, R., \& Ullash, S. (2020). Detecting and Healing Area Coverages Holes in homogeneous Wireless Sensor Network: Survey. arXiv preprint arXiv:2005.02492.

[41] Kashi, S. S. (2019). Area coverage of heterogeneous wireless sensor networks in support of Internet of Things demands. Computing, 101(4), 363-385.

[42] Li, Q., \& Liu, N. (2020). Monitoring area coverage optimization algorithm based on nodes perceptual mathematical model in wireless sensor networks. Computer Communications, 155, 227234.

[43] M. Sfendourakis, N. Rajagopal, Emm. Antonidakis "Triangulation positioning system network" 359 MATEC Web Conf. 12502069 (2017) DOI: 10.1051/matecconf/201712502069.

\section{Creative Commons Attribution License 4.0 (Attribution 4.0 International, CC BY 4.0)}

This article is published under the terms of the Creative Commons Attribution License 4.0

https://creativecommons.org/licenses/by/4.0/deed.en_US 